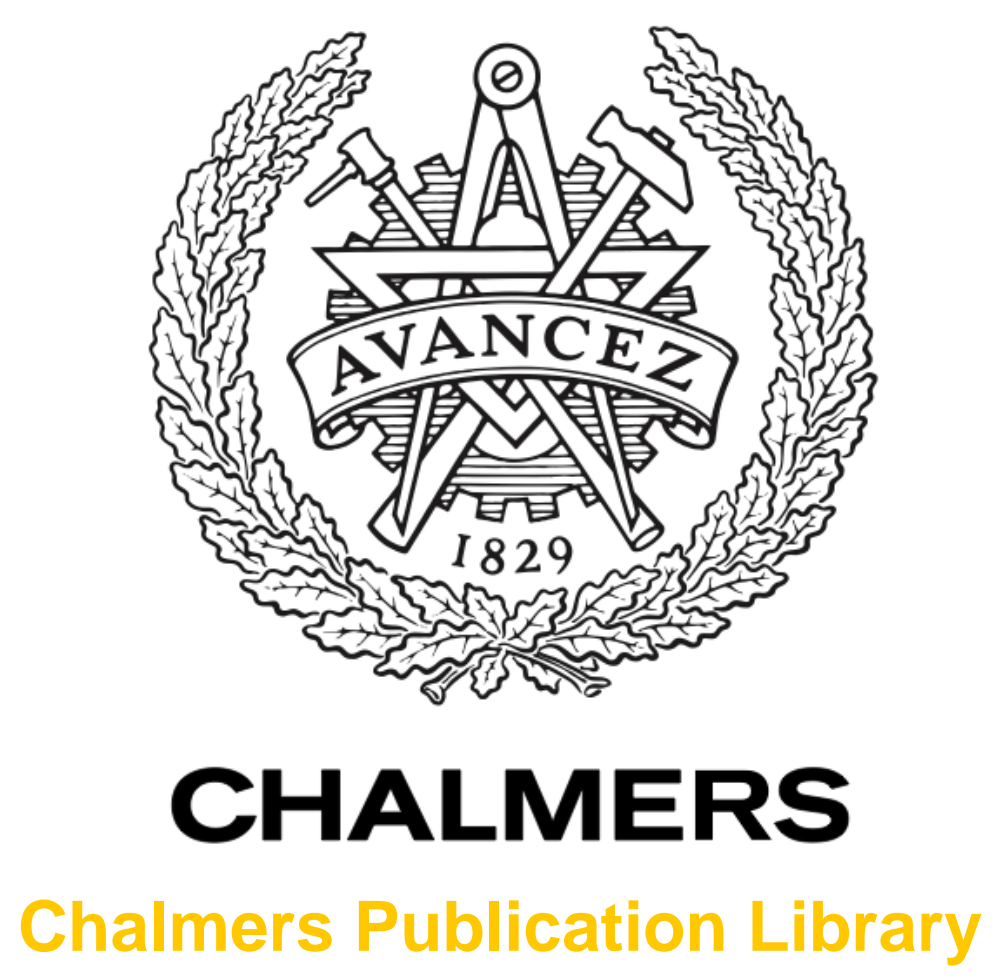

\title{
Pull-out of textile reinforcement in concrete
}

This document has been downloaded from Chalmers Publication Library (CPL). It is the author's version of a work that was accepted for publication in:

\section{Construction and Building Materials (ISSN: 0950-0618)}

Citation for the published paper:

Williams Portal, N. ; Perez, I. ; Thrane, L. et al. (2014) "Pull-out of textile reinforcement in concrete". Construction and Building Materials, vol. 71 pp. 63-71.

http://dx.doi.org/10.1016/j.conbuildmat.2014.08.01

Down「oaded from: http://publications.lib.chalmers.se/publication/209225

Notice: Changes introduced as a result of publishing processes such as copy-editing and formatting may not be reflected in this document. For a definitive version of this work, please refer to the published source. Please note that access to the published version might require a subscription. 


\title{
Pull-out of Textile Reinforcement in Concrete
}

Natalie Williams Portal, M.Sc. ${ }^{1}$, Ignasi Fernandez Perez, M.Sc. ${ }^{2}$

Lars Nyholm Thrane, Ph.D. ${ }^{3}$, Karin Lundgren, Professor ${ }^{4}$

${ }^{1}$ Dept. of Civil and Environmental Engineering, Chalmers University of Technology, Gothenburg 412 96, Sweden, E-mail: Natalie.williams@chalmers.se

${ }^{2}$ Dept. of Construction Engineering, Universitat Politècnica de Catalunya - Barcelona Tech, Barcelona, 08034, Spain, E-mail: Ignasi.fernandez-perez@upc.edu

${ }^{3}$ Dept. of Building Technology - Concrete, Danish Technological Institute, Taastrup 2630, Denmark, E-mail: Inth@dti.dk

${ }^{4}$ Dept. of Civil and Environmental Engineering, Chalmers University of Technology, Gothenburg 412 96, Sweden, E-mail: Karin.lundgren@chalmers.se

\section{Highlights:}

- Pull-out tests of TRC to gain better knowledge of the complex bond behaviour.

- Local-bond slip relationship evaluated from experimental data.

- $1 \mathrm{D}$ and 3D numerical models to simulate complex global bond behaviour.

- 3D models validated the simplified assumptions applied in the 1D model.

\begin{abstract}
:
Textile Reinforced Concrete (TRC) has emerged as a promising novel alternative offering corrosion resistance and both thinner and light-weight structures. Although TRC has been extensively researched, the formalization of experimental methods and design standards is still in progress. The aim of this work was to extract local-bond behaviour from pull-out tests of basalt and carbon TRC and utilize these in both simple (1D) and advanced models (3D) to yield the global structural behaviour. The simulation results from the $1 \mathrm{D}$ and $3 \mathrm{D}$ models are able to simulate the complex behaviour of TRC with a reasonable level of correlation.
\end{abstract}

Keywords: Textile reinforced concrete (TRC); Bond-slip; Pull-out tests; Experimental tests, Finite-element modelling 


\section{Introduction}

A recent innovative attempt to improve the sustainability of reinforced concrete is the development of Textile Reinforced Concrete (TRC) encompassing a fine-grained concrete matrix reinforced by a multi-axial non-corrosive textile mesh. This relatively new composite material has been extensively researched at collaborative research centres 532 and 528 at RWTH Aachen University and Dresden University of Technology [1] over the past decade. Collaborative efforts spreading across USA, Germany, Brazil and Israel have also played a major role in this field [2]. It was discovered that TRC can be utilized to build slender, lightweight, modular and freeform structures and eliminate the risk of corrosion. The completion of a pedestrian bridge fabricated solely of TRC [3] and the development of thin self-supporting TRC sandwich elements [4] are examples of the possible realizations. It was also proven to be an adequate strengthening material for existing reinforced concrete structures in a variety of applications [5, 6]. To sum up, a report entitled RILEM TC 232-TDT encompassing test methods and design of Textile Reinforced Concrete, in progress since 2009, has been compiled, however is not yet available for public use [7]. The purpose of this particular report is to provide guidelines for testing methods, a design manual and an update of the TRC state-of-the-art report [8].

In fibre composite materials, such as TRC, bond behaviour between the yarn or roving and the cementitious matrix is a principal factor influencing the global structural behaviour [9]. Yarns or rovings consist of multitudes of filaments which creates a complex heterogeneous structure. For that reason, the characterization of the bond behaviour is critical in terms of input for numerical models analysing the structural behaviour of TRC. Pull-out testing is a typical method utilized to gain understanding of bond phenomenon related to reinforced concrete. Since the lack of standards in this field, testing methods and numerical methods to evaluate the pull-out behaviour of yarns or rovings in TRC used in similar research need to be explored and considered. Bond properties of TRC have been investigated using various textile pull-out test configurations while focusing on differing textile meshes and influential parameters by Krüger [10], Xu and Li [11], Sueki, Soranakom et al [12], Ortlepp, Curbach et al [13], Lorenz and Ortlepp [14] and Zhang, Aljewifi et al [15]. For instance, in [12], a parametric study of varying embedment lengths, textile meshes and cement mix types as well as processing methods were incorporated. Specimens reinforced by alkali-resistant (AR) glass, polypropylene and polyvinyl acetate meshes were prepared using casting, pultrusion or vacuum techniques. Successively, the use of analytical methods followed the experiments of the abovementioned works such that the experimental pullout behaviour of a single continuous yarn from a matrix was described analytically using closed 
form equations [9, 12, 14-16]. Various damage models such as a triple linear shear-stress slip model have been assumed to characterize the yarn pull-out behaviour incorporating both adhesion and frictional load transfer $[9,16]$. In brief, experimental results from pull-out tests appear to successfully verify the developed analytical and numerical models $[9,12,14-16]$.

Direct pull-out tests are included in this research to characterize the complex pull-out behaviour of a textile mesh-structure embedded in a concrete matrix with a particular focus on basalt and carbon TRC. More specifically, the pull-out of a single roving from the textile mesh was carried out which resulted in a representative smeared pull-out behaviour of the embedded mesh structure. Varying embedment lengths were used to quantify bond capacity and textile rupture failure modes. Based on the experimental results, a local bond stress-slip curve was obtained. Thereafter, the calibrated local behaviour was used as material input data in two numerical models to simulate the global behaviour experienced by the pull-out specimens. An analytical 1D bond model originally developed by Lundgren, Kettil et al [17] to analyse the bond stress-slip behaviour of corroded ribbed steel reinforcement was modified for textile reinforcement. Also, in the 1D model, a stepwise calculation and superposition is needed to obtain the bond stress-slip behaviour for the entire pull-out specimen. Moreover, a 3D non-linear finite element model of the conducted pull-out tests was developed and used to validate the assumptions related to the simplest 1D bond model. Using simplified models to study bond capacity of TRC concrete is necessary to better understand complex structural behaviour.

\section{Experimental study}

The presented work is a part of a larger experimental scope, primarily conducted at the Danish Technological Institute (DTI) and evaluated at Chalmers, which encompassed flexural and pullout tests of TRC. The experimental work presented here focuses on direct pull-out tests of TRC specimens reinforced by basalt and carbon meshes having the underlying purpose to characterize the corresponding bond phenomenon. The mechanical properties of the cementitious matrix were also obtained through compressive and tensile splitting tests.

\subsection{Pull-out testing}

At present, no standard test setup to investigate the pull-out behaviour of TRC is available; as such, relevant experimental work from literature was revealed to help establish an experimental setup. The main types of test configurations known from literature include one-sided and doublesided pull-out tests. For example, one-sided tests were conducted by Banholzer [18], wherein 
single yarns were embedded in a concrete matrix at one end and in an epoxy resin block at the other. This type of test is, however, said to yield a behaviour that is incomparable of that of a textile mesh [14]. As for two-sided tests, either symmetrical [19] or unsymmetrical anchoring lengths [10] can be implemented. The benefit of using unsymmetrical anchoring lengths is that a longer length guaranteeing adequate anchoring of the yarn/roving can be incorporated into the setup [14].

The pull-out test setup and specimen configuration employed in this work was designed based on the double-sided unsymmetrical test by Krüger [10] and Lorenz and Ortlepp [14]. The pull-out test specimens used in the presented experimental work measured $400 \times 100 \times 15 \mathrm{~mm}$ and reinforced by one layer of reinforcement mesh. Unsymmetrical anchorage lengths, denoted $A$ and $B$ in Fig. 1, were defined for each specimen. A singular roving from the textile mesh was exclusively tested from the TRC specimen in order to yield representative smeared pull-out behaviour. The embedment length chosen was generally based on the distance of the crossthreads, specified as Short (35 mm), Medium $(70 \mathrm{~mm})$ and Long $(87.5 \mathrm{~mm})$ for basalt and Short (25 mm), Medium $(50 \mathrm{~mm}$ ) and Long $(75 \mathrm{~mm})$ for carbon. The prescribed embedded length was limited to the upper end of the specimen by means of a single saw cut crossing the roving to be tested and a breaking point marked by two saw cuts isolating the examined roving. The breaking point does not designate the definitive breaking point of the roving in itself, but rather the location of crack initiation. 

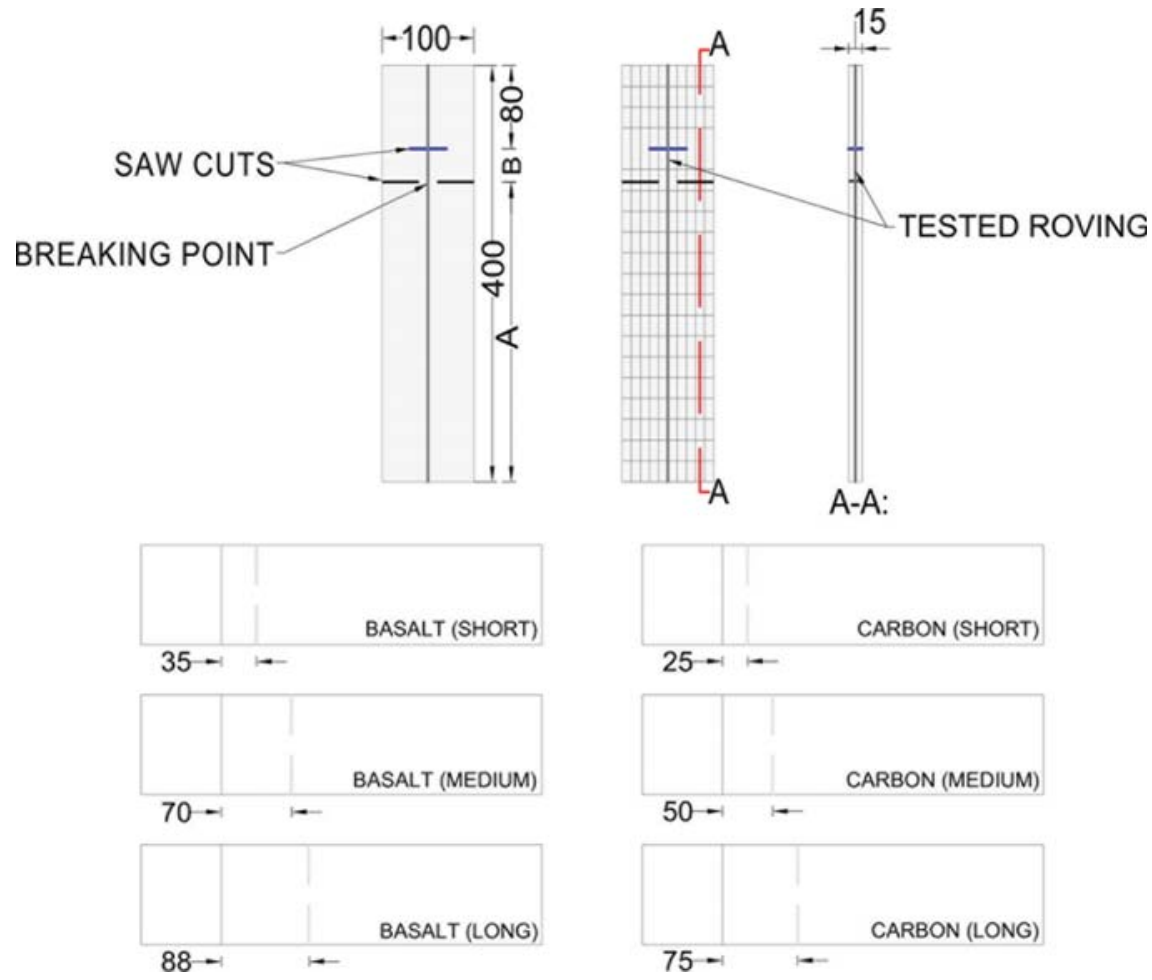

Fig. 1. Test specimen configuration and embedment length $(\mathrm{mm})$.

The pull-out tests encompassed the evaluation of varying embedment lengths in order to characterize both pull-out and rupture of the textile roving as failure modes. Up to three specimens were produced for each selected embedment length in order to obtain a representative trend of the pull-out behaviour. The pull-out test specimens were configured such that the rovings along the pull-out direction later shown in Fig. 3 were positioned in the direction of the machine pull-out force (see Fig. 2). The layer of textile mesh was fastened by the framework used to cast the specimens causing the mesh to become slightly taut yet not prestressed which could have slightly reduced the initial waviness of the mesh. As per [1], prestressed TRC members exhibit higher peak loads at the expense of lower slip and a more brittle behaviour. 

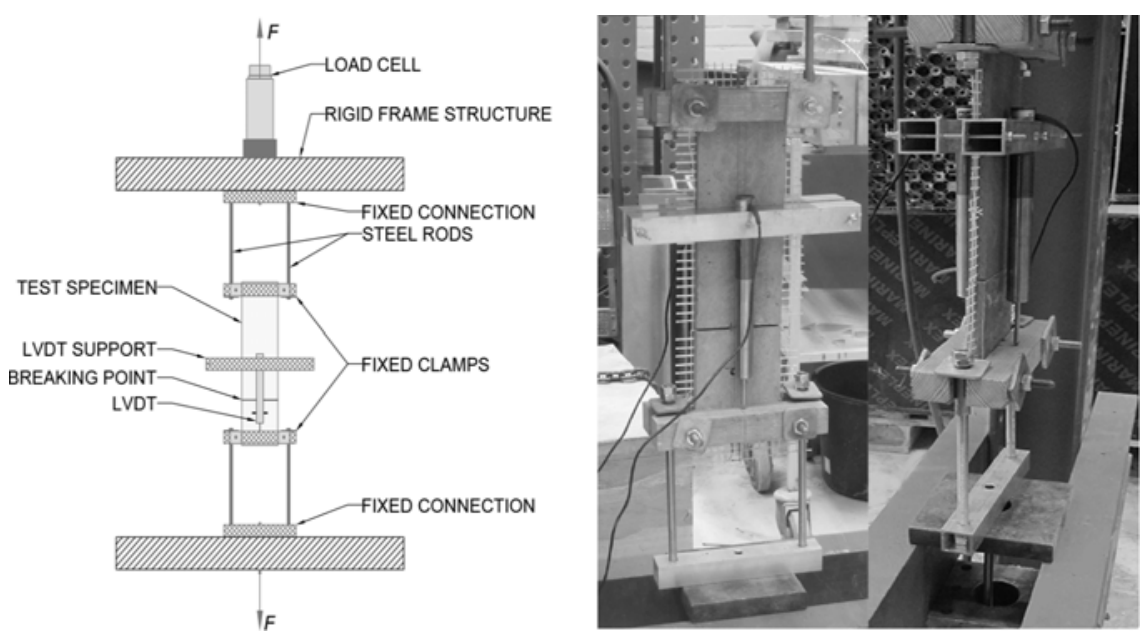

Fig. 2. Sketch (left) and photo (right) of experimental test setup.

The experimental setup developed to conduct the pull-out tests is illustrated in Fig. 2. The load was applied by a hydraulic jack on top of the rigid frame structure. On top of the hydraulic jack, a $25 \mathrm{kN}$ load cell was placed in order to measure the load. The ends of the test specimen were affixed by two wood clamps which were used to transfer the load to the specimen. The load was transferred symmetrically to the wood clamps by means of steel rods on both sides of the specimen which were further linked to connections fixed to the rigid frame structure. The total specimen deformation, i.e. crack-opening relationship at the breaking point, of the test specimen was measured using two linear variable displacement transducers (LVDT) positioned on either side of the centre of the specimen. Load and deformations were measured and stored every second by means of a data logger.

\subsection{Material description}

The pull-out test specimens were fabricated of a fine-grained concrete matrix according to the mix composition stated in Table 1.The mean concrete cylinder compressive strength corresponding to 28 days, $f_{c m}$, was derived from material test results to be $53.6 \mathrm{MPa}$. Based on $f_{c m}$, the mean modulus of elasticity, $E_{c m}$, was estimated to be $36.4 \mathrm{GPa}$ using CEN [20]. Lastly, the mean value of the tensile splitting strength tests was found to be $4.7 \mathrm{MPa}$. 
Table 1. Composition of the fine-grained concrete, mass in $\mathrm{kg}$ per $\mathrm{m}^{3}$ of concrete.

\begin{tabular}{lll}
\hline Matrix composition & Density $\left[\mathrm{kg} / \mathrm{m}^{3}\right]$ & Quantity $\left[\mathrm{kg} / \mathrm{m}^{3}\right]$ \\
\hline Low alkali cement (Z 52.5) & 3200 & 406.0 \\
Fly ash & 2300 & 121.0 \\
Microsilica & 2200 & 22.0 \\
Glenium SKY 532 - SU & 1100 & 7.6 \\
Amex SB22 (air entrainer) & 1010 & 3.0 \\
O/4 Sand & 2640 & 1400.0 \\
Water & 1000 & 170.6 \\
\hline
\end{tabular}

Two different types of textile meshes fabricated of carbon and basalt were included in this study. The carbon mesh used was produced by SGL group (Germany) is SIGRATEX grid 300 with a mesh spacing of $30 \times 30 \mathrm{~mm}$ with roving fineness of 1600 tex. Lastly, Geo-grid mesh (Zhejiang GBF, China) fabricated of basalt with silane sizing. It has a configuration of $25 \times 25 \mathrm{~mm}$ with a roving fineness of 2000 tex. The geometry, configuration of the cross-threads and pull-out direction pertaining to these textile meshes are depicted in Fig. 3. As well, corresponding nominal material properties from the manufacturers are listed in Table 2.

Table 2. Material properties for textile reinforcement alternatives.

\begin{tabular}{lllll}
\hline Material & $\begin{array}{l}\text { Roving cross- } \\
\text { sectional area }\left[\mathrm{m}^{2}\right]\end{array}$ & $\begin{array}{l}\text { Specific surface } \\
\text { weight }\left[\mathrm{g} / \mathrm{m}^{2}\right]\end{array}$ & $\begin{array}{l}\text { Tensile strength } \\
{[\mathrm{MPa}]}\end{array}$ & $\begin{array}{l}\text { Young's } \\
\text { Modulus [GPa] }\end{array}$ \\
\hline Basalt & $0.755 \times 10^{-6}$ & 303 & 1898 & 100 \\
Carbon & $0.889 \times 10^{-6}$ & 300 & 3800 & 230 \\
\hline
\end{tabular}

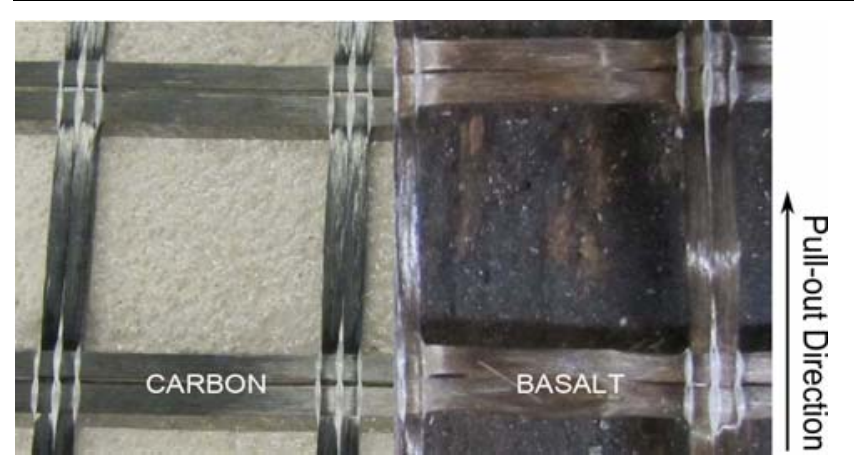

Fig. 3. Overview of the textile meshes.

\subsection{Result summary}

\subsubsection{Basalt}

The force versus total deformation trend corresponding to the basalt specimens for all embedment lengths are described in Fig. 4. The total displacement corresponds to the mean 
displacement recorded by the two LVDTs for the entire specimen. In Fig. 4, it is observed that as the embedment length increases, the maximum force increases and occurs at a larger deformation. A pull-out failure mode was solely yielded for Short specimens, while rupture was the common failure mode for both Medium and Long specimens. In the case of pull-out failure, the pre-peak bond behaviour is governed by adhesive bond which is followed by the destruction of the adhesive bond occurring due to debonding of the roving from the matrix. Lastly, the remaining pull-out force is based on friction, as described in [16]. As can be seen in Fig. 4, there was, however, especially for the tests with embedment length $87.5 \mathrm{~mm}$, a rather long plateau with roughly constant load, before a sudden loss of capacity at rupture of the textile roving.

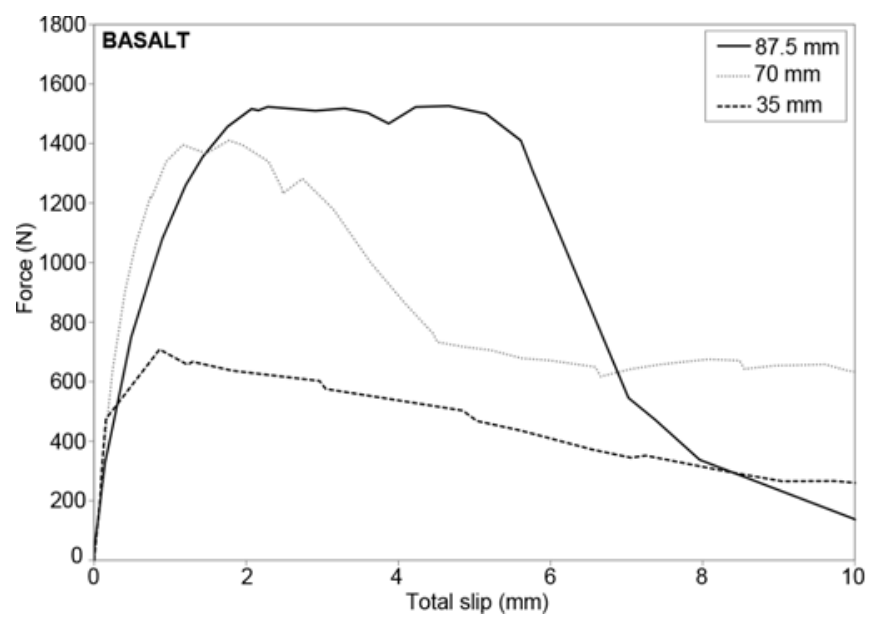

Fig. 4. Basalt average experimental results (all embedment lengths).

The average maximum force corresponding to Short specimens was $721 \mathrm{~N}(\sigma=79), 1423 \mathrm{~N}$ $(\sigma=63)$ for Medium and $1662 \mathrm{~N}(\sigma=139)$ for Long. Causes of variability in the results are presumed to be: uneven bond penetration through cross-section of roving, potential bond irregularities along embedment length (weak zones), human error in sample preparation as well as limited experimental sample size.

\subsubsection{Carbon}

Fig. 5 depicts the force versus total deformation trend for the carbon specimens for all embedment lengths. Similarly to basalt, the maximum force increases and the related deformation also slightly increases as the embedment length gets larger. The maximum force corresponding to Short specimens was $629 \mathrm{~N}(\sigma=32), 1050 \mathrm{~N}(\sigma=154)$ for Medium specimens and $1333 \mathrm{~N}(\sigma=298)$ for Long specimens. Moreover, variability in the presented results is likely due to the similar aforementioned reasons related to the basalt specimens. 


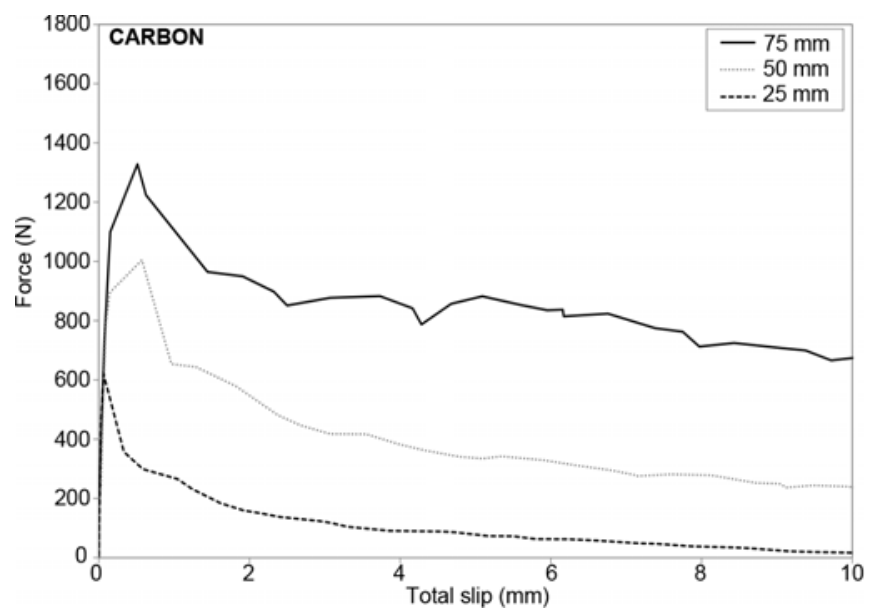

Fig. 5. Carbon average experimental results (all embedment lengths).

The common failure mode for the carbon textile roving was marked by pull-out of the roving in this study. It should be noted that an uneven pull-out of the roving was observed, presumably due to the heterogeneous bond existing between the roving structure and matrix. The mechanical description of the pull-out phenomenon is thought to be similar to that previously explained for basalt. Furthermore, it is believed that rupture failure was inhibited by the surface smoothness of the uncoated fibres as well as insufficient embedment length. Likewise, Lorenz and Ortlepp [21] demonstrated that to yield rupture of the roving, uncoated carbon textile rovings requires an embedment length of $80 \mathrm{~mm}$ which is $50 \%$ greater than needed for the same textile roving with epoxy coating.

\section{Local Bond Stress-Slip Curve}

The shape of the local bond stress-slip function depends on concrete material properties (i.e. compressive strength), textile roving geometry (i.e. perimeter) as well as the configuration of the mesh cross-threads. The local bond stress-slip function can typically be estimated using various numerical [22] or analytical methods [9, 12, 14]. In this study, the first estimate of a local bond stress-slip function was obtained from the experimental results (force versus total deformation) from the Short specimens (refer to Fig. 1). The typical assumption for short pull-out tests with ribbed steel reinforcement bars, defined by embedment length of less than five times the bar diameter, is that the distribution of bond stresses is uniform along the reinforcement [23, 24]. Concerning textile reinforcement it is difficult to foresee that this simplified condition could apply due to the heterogeneous behaviour of the roving, nevertheless it was tested and evaluated in 
this study. Accordingly, the uniform bond stresses hypothesis was assumed for the Short embedded length specimens, such that the general bond stress could be estimated as:

(1) $T=P / \pi \Phi L$

where, $P$ is the load, $\Phi$ is the nominal diameter of the roving; and $L$ is the embedded length. Initially, for the Short specimen, it was assumed that the slip was entirely taken by the Short zone before and after the peak. Since this hypothesis underestimates first bond stress values for the Short zone, the initial estimation of the local bond-slip was modified, particularly in the prepeak region, until a reasonable fit for all embedment lengths was obtained, as further described in Section 4. Due to the performed pull-out test and type of method used to record the slip, the non-linearity of the post-peak region was not adequately characterized. However, within this region, the slip distribution can be assumed to be entirely taken by the Short zone (refer to Section 4). The calibration of the bond stress-slip curve was accomplished by a power function in the pre-peak region, i.e. from zero to the slip at ultimate bond stress, $s\left(\mathrm{~T}_{\mathrm{ult}}\right)$, and by a linear functions in the post-peak region, i.e. from the slip at ultimate bond stress, $\mathrm{s}\left(\mathrm{T}_{\mathrm{ult}}\right)$, to the ultimate slip, $\mathrm{s}_{\mathrm{ult}}$, as per Eq. 2. This calibration method is similar to that proposed in the fib Bulletin 55 : Model Code 2010 for bonded FRP rebars [22].

(2) $T(S)=T_{\text {ult }}\left(S / s_{\text {ult }}\right)^{B} \in 0 \leq s \leq S\left(T_{\text {ult }}\right)$, Power function - Basalt

$T(S)=-T_{\text {ult }}\left(1-S / s_{\text {ult }}\right)^{B}+T_{\text {ult }} \in 0 \leq \mathrm{S} \leq S\left(T_{\text {ult }}\right)$, Power function - Carbon

$T(s)=m \cdot s+b \quad \in \mathrm{S}\left(T_{\text {ult }}\right) \leq \mathrm{s} \leq \mathrm{s}_{\text {ult }}$, Linear function - All

where, $\mathrm{s}$ is a given slip value, $B$ is the weighting factor; and $m$ and $\mathrm{b}$ are the corresponding slope and $y$-intercept of the linear function. The calibrated local bond-slip curves for basalt and carbon TRC are shown in Fig. 6 together with the first estimate obtained as described above. The calibrated bond-slip curves described in Table 3 were subsequently used as input in non-linear finite element modelling to simulate the global behaviour of the pull-out specimens.
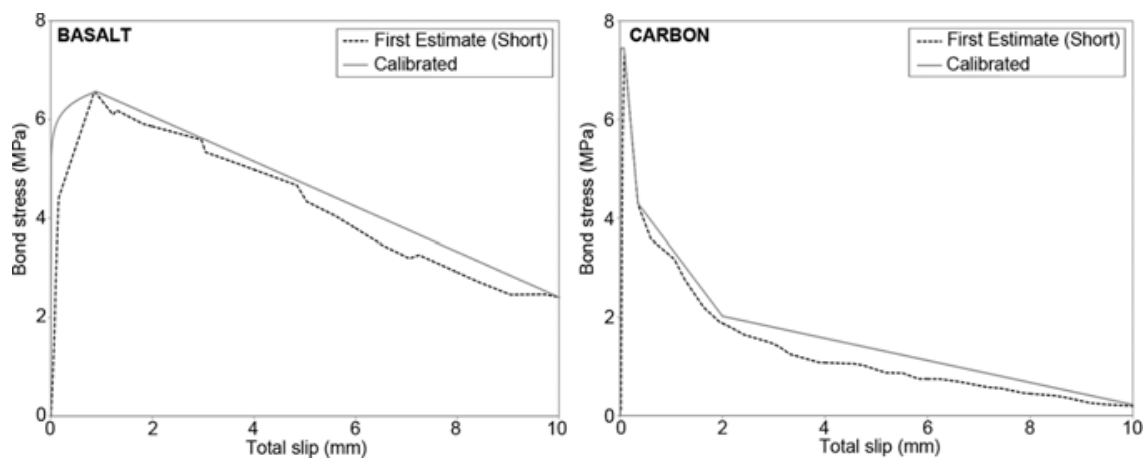
Fig. 6. Calibrated local bond-slip for basalt (left) and carbon (right).

Table 3. Input for the calibration of local bond-slip curves

\begin{tabular}{llll}
\hline Material & $\begin{array}{l}\text { Power function } \\
\text { B, weighting factor }\end{array}$ & $\begin{array}{l}\text { Linear function } \\
\mathrm{m} \text {, slope } \\
\left(\mathrm{N} / \mathrm{mm}^{3}\right)\end{array}$ & $\begin{array}{l}\text { Linear function } \\
\text { b, } \mathrm{y} \text {-intercept } \\
\left(\mathrm{N} / \mathrm{mm}^{2}\right)\end{array}$ \\
\hline Basalt & 0.05 & -0.46 & 6.98 \\
Carbon & 100 & -11.67 & 8.38 \\
& & -1.38 & 4.78 \\
& & -0.22 & 2.47 \\
\hline
\end{tabular}

\section{Numerical Modelling}

A 1D model was used in this work to simulate the bond behaviour observed in the experimental study. The assumptions and limitations of this presented model were validated using a 3D nonlinear model developed in the finite element analysis commercial software DIANA (DIsplacement ANAlyser) with pre- and post-processor Midas FX+.

\subsection{D Bond model}

An analytical 1D bond-slip model developed by [17], principally used to analyse the bond stressslip behaviour of corroded and uncorroded ribbed steel reinforcement, was modified for textile reinforcement in this study. To commence, the steel reinforcement is replaced by a singular continuous textile roving assumed to be homogeneous with a circular cross-section wherein the transverse rovings of the mesh are excluded. The stress and strain distribution for the conducted pull-out test can be described using Fig. 7. Accordingly, the differential equation expressing equilibrium conditions along the reinforcement in tension can be defined as Eq. 3:

(3) $\pi \Phi^{2} / 4 \cdot d \sigma_{t} / d x-\pi \Phi_{T}=0$

where, $\phi$ is the reinforcement diameter, $\sigma_{t}$ is the stress in the textile reinforcement and $t$ is the bond stress. The model was developed to analyse the bond stress-slip behaviour of the equivalent textile roving within the so-called anchorage length, thus the stress in the reinforcement is assumed to be in the elastic range according to Eq. 4:

(4) $\sigma_{t}=E_{t} \varepsilon_{t}, \varepsilon_{t}=d u / d x$

where, $E$ is Young's modulus, $\varepsilon$ is the strain and $u$ is the displacement of the reinforcement. Also, in this model, the bond stress follows an elasto-plastic law implying that the deformation of the surrounding concrete is assumed to be negligible; thereby the displacement of the 
reinforcement is equal to the slip. Furthermore, to solve the equilibrium equation stated in Eq. 3, boundary conditions based on the pull-out of an equivalent textile roving having a length of $L$ and a prescribed displacement $u_{L}$ were defined as per Eq. 5 :

(5) $\sigma_{t}(0)=0, u(L)=u_{L}$

The response of the pull-out tests were computed using a differential equation solver in MATLAB [25]. For further details regarding the development and implementation of the 1D bond-slip model refer to Lundgren, Kettil [17]. Moreover, to determine the total slip from the 1D bond-slip model, the slip contributions from the Short and Long zones were added to result in the total slip distribution which could thereafter be compared to the experimental results. The slip from the Long zone includes an assumed linear-elastic recovery curve to zero slip after the peak force. The corresponding results are presented and evaluated in Section 5.
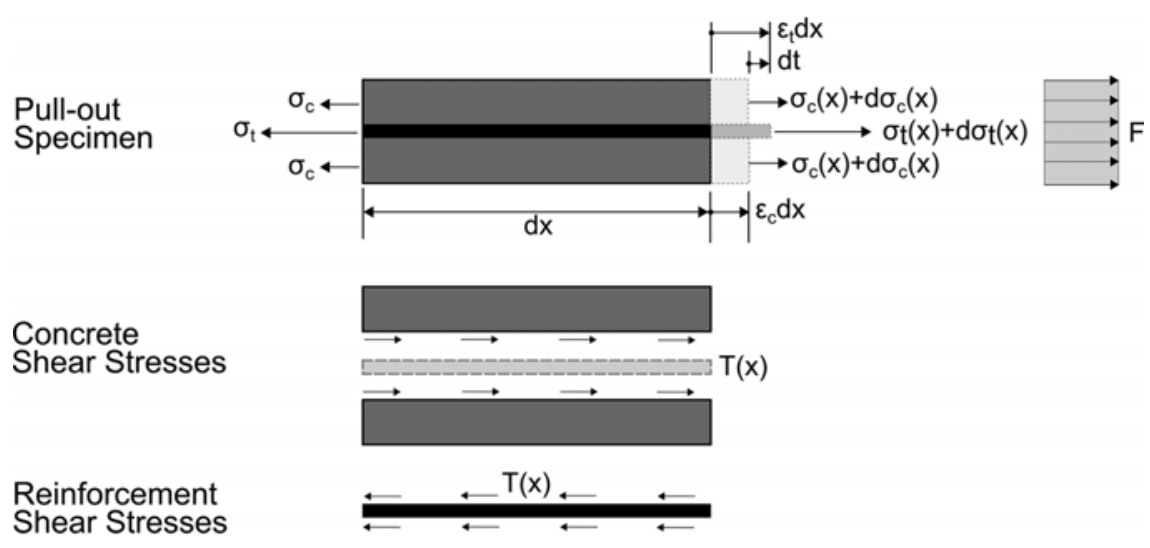

Fig. 7. Distribution of stresses and strains of the pull-out test; where subscript $c$ and $t$ denotes concrete and textile roving stresses or strains, respectively.

\subsection{Non-linear FE analysis}

3D non-linear finite element analyses were performed to describe the non-linear behaviour of the bond-slip relationship between concrete and the textile reinforcement mesh. The verification of the model was accomplished by means of the experimental results obtained from the pull-out tests carried out in the laboratory. The results were also used to validate the assumptions included in the above-mentioned analytical 1D bond-slip model presented in Section 4.1. DIANA with pre- and post-processor Midas FX+ was used for the numerical simulations [26]. The pullout specimens that were modelled had the same dimensions as the tested specimens, incorporated the different embedded lengths prescribed based on the material and included exclusively a singular roving. 


\subsubsection{Load and boundary conditions}

The idealized pull-out specimen, shown in Fig. 8 was loaded until bond failure similarly as in the laboratory conditions. Concerning the Long zone of the specimen, fixed supports were considered in three principal directions at the end side. The load was applied onto the Short zone by means of displacement, such that an imposed displacement was uniformly applied over the entire end surface. Additional supports were defined in some points along the specimen as a mesh stabilizer to avoid displacements in other directions as well as torsion. The analysis was carried out using a stepwise non-linear analysis with controlled displacement of the Short zone.

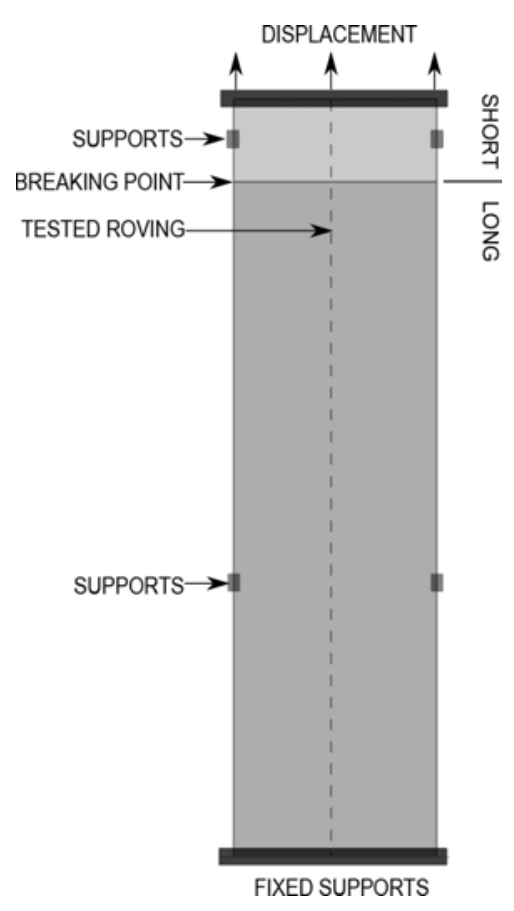

Fig. 8. Idealized 3D model of pull-out test.

\subsubsection{Element types \& materials}

The model, illustrated in Fig. 9, included four-node 3D isoparametric solid pyramid linear elements for concrete entitled TE12L in DIANA, embedded reinforcement for the textile and interface elements between the specimen's contact planes in a $3 \mathrm{D}$ configuration. Interface elements were used to make the connection of the two bodies of the specimens (Short and Long) possible and to be able to use embedded reinforcement for the textile roving with an assigned calibrated bond-slip relationship. The interface layer was defined as a discrete crack layer without tensile strength. Furthermore, tyings were defined at the prescribed pull location which ensures an even displacement along the surface where the clamps were acting. 
Essentially, all the nodes of this surface were tied to the centre node which was loaded by the prescribed displacement.

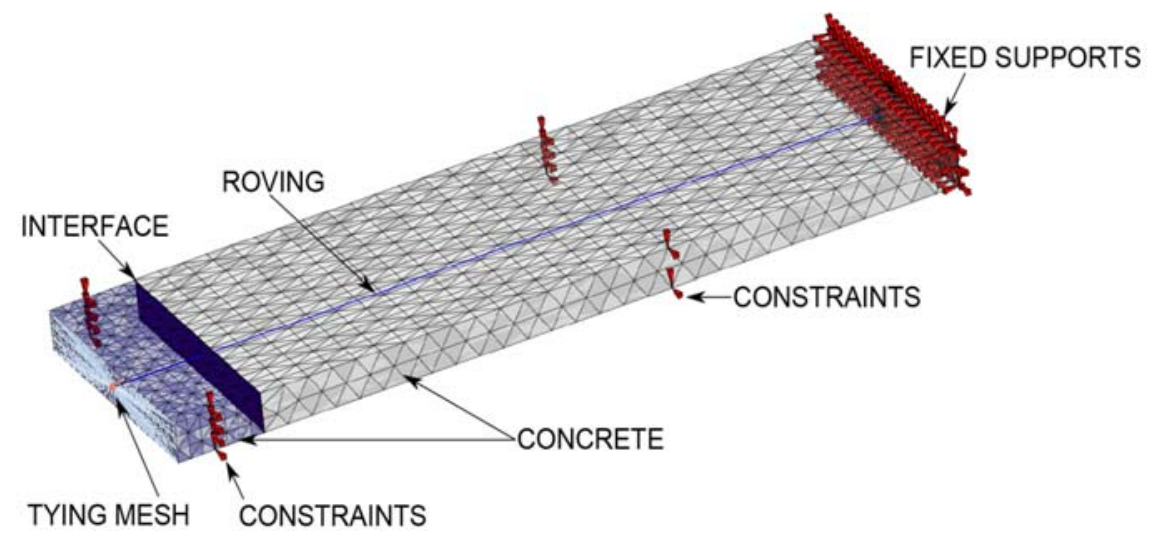

Fig. 9. Overview of 3D model in DIANA.

The element size was chosen ranging from 5 to $10 \mathrm{~mm}$, regarded as a fine mesh, in order to obtain sufficient intersection points between the embedded textile reinforcement and the mesh, as well as to properly characterize the bond stresses along the textile roving. Material properties for both concrete and textile were chosen as linear-elastic, which corresponds to the outcome of the experiment. The tensile stress range for concrete was found to be under the corresponding tensile strength and the textile being a brittle material, has a linear- elastic behaviour until failure. However, the designed bond-slip relationship between both materials was chosen as non-linear to reproduce the behaviour of the tested specimens and to be able to compare with the $1 \mathrm{D}$ analysis results. The calibrated bond-slip relationship used in the model was identical to that used in the 1D model as previously described. The mechanical properties of both the concrete and textile reinforcement were obtained either based on experimental results or derived from manufacturer data, as aforementioned in Section 2.2.

\section{Comparison and discussion}

The slip distributions of Short and Long zones versus total slip are shown in Fig. 10 (left) for basalt and Fig. 11 (left) for carbon. The experimental and numerical results for all embedment lengths compared in Fig. 10 (right) for basalt and Fig. 11 (right) for carbon depict a reasonably good correlation. The 1D model generally simulates the stiffness prior to the peak and the maximum force with reasonable good agreement. As for post-peak behaviour, the rupture of the roving causing a sudden drop in stiffness is not captured using the 1D model for the Medium and Long specimens because of the linear elastic material assumption. 
The 3D models were used to evaluate the scope of the 1D model related to basalt as well as to validate the different assumptions. The concrete stress/strain distribution in the $3 \mathrm{D}$ analysis results confirm that the contribution of the deformation of the roving to the slip is negligible (see Fig. 12), thus validating the main assumption of the $1 \mathrm{D}$ model. Furthermore, the 3D model was able to capture the variation of bond stresses occurring during post-peak loading along the Short and Long zones because it reproduces the interaction between both parts and includes unloading. Fig. 13 depicts that the bond stress distribution for the short embedment length was non-uniform for the initial slip values (i.e. 0.01-0.3 mm). Therefore, the local-bond values in the first estimate from the experimental data were initially underestimated and adjustment was necessary particularly in the pre-peak region to take into account the non-uniform bond stress distribution.
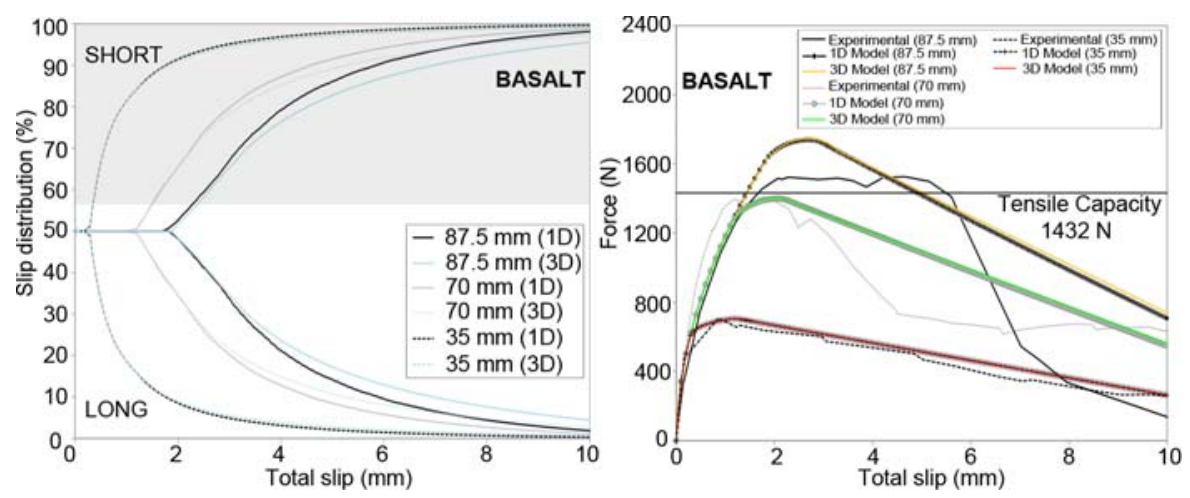

Fig. 10. Slip distributions of Short and Long zones versus total slip (left) and experimental results versus $1 \mathrm{D}$ and $3 \mathrm{D}$ model for basalt (right).
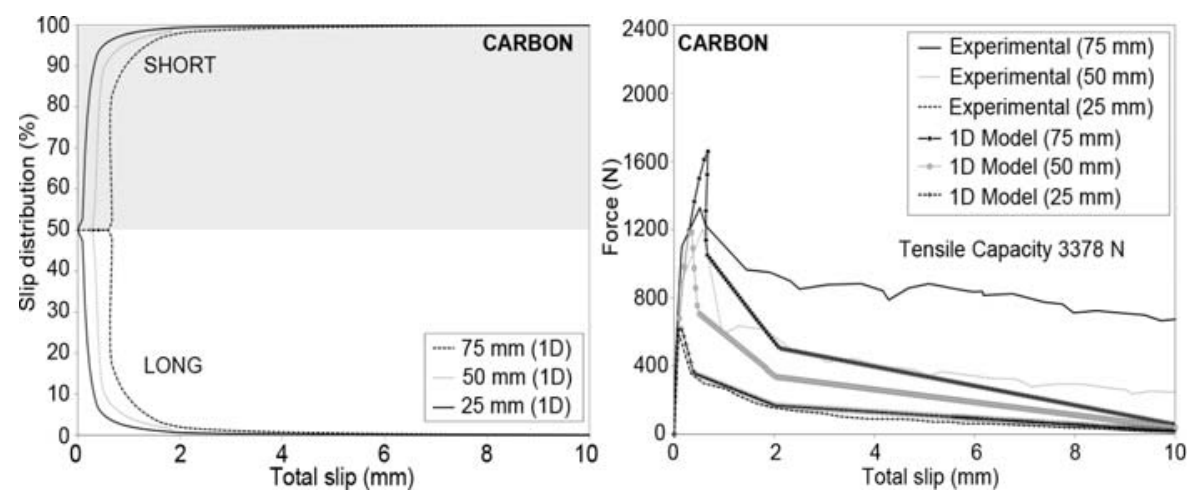

Fig. 11. Slip distributions of Short and Long zones versus total slip (left) and experimental results versus 1D model for carbon (right). 


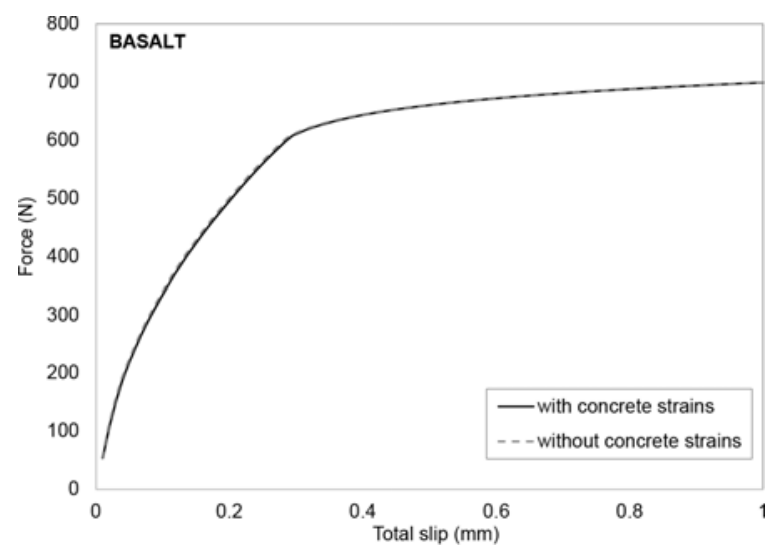

Fig. 12. Total deformation with and without concrete strains simulated by 3D model for basalt Short specimens.

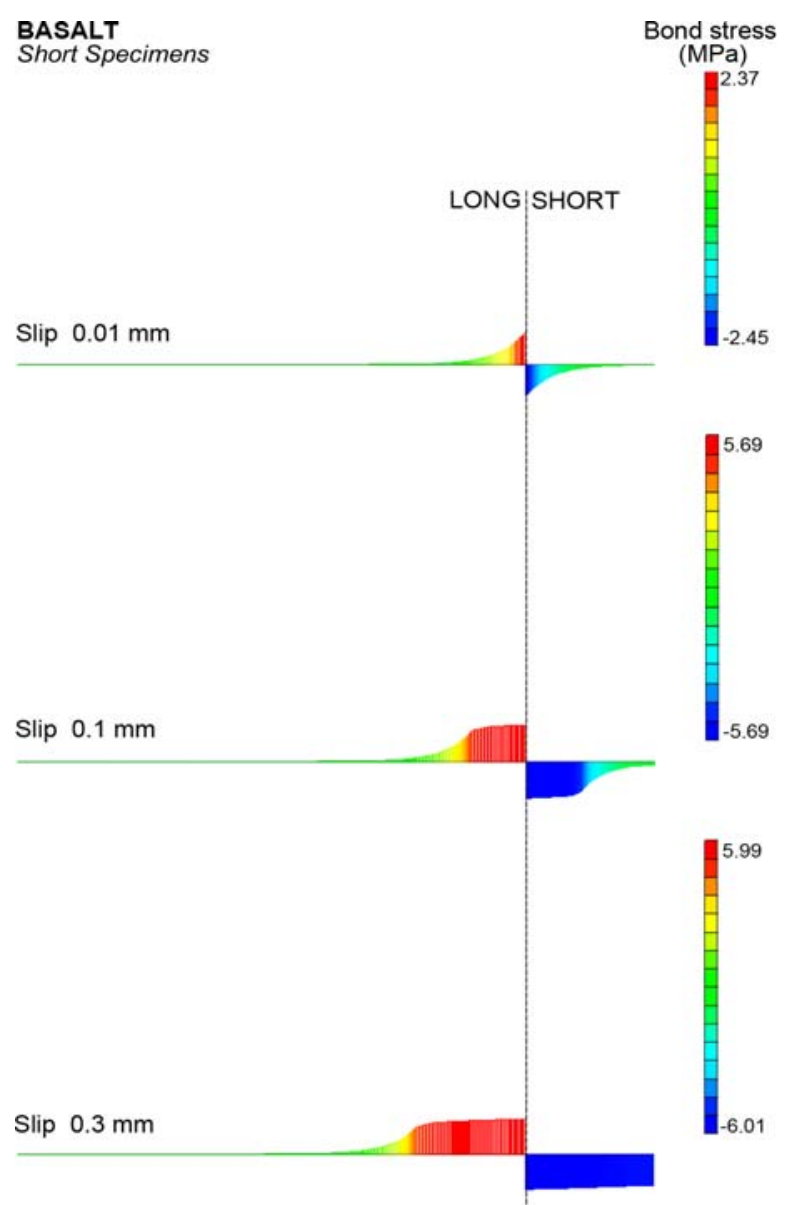

Fig. 13. Bond stress evolution simulated by 3D model for basalt Short specimens at different load steps corresponding to various slips $(0.01 \mathrm{~mm}, 0.1 \mathrm{~mm}$ and $0.3 \mathrm{~mm})$. 


\section{Conclusions}

The bond behaviour of basalt textile reinforced concrete was characterized in this work by means of direct pull-out tests. Pull-out failure was observed for Short specimens, while rupture failure was noted for Medium and Long in the experimental pull-out tests for basalt textile reinforced concrete specimens. As for carbon, pull-out failure was the common failure mode experienced by all specimens. Thereafter, a local bond stress-slip curve was calibrated for the basalt and carbon specimens based on the experimental results related to the Short specimens. Moreover, applying this local bond stress-slip curve in a simple 1D bond model demonstrated a reasonable force versus total displacement correlation with the experimental results for the longer embedment lengths as well. The pull-out test setup presented in this work is suitable to obtain and characterize local bond behaviour for different TRC materials, particularly in the case of short embedment lengths. Non-linear 3D models proved that the assumptions made to obtain the adjusted local bond-slip behaviour are acceptable and capture a sufficient amount of accuracy. Also, these models were valuable tools which validated the different hypothesis made in the simpler 1D model used to describe the bond behaviour of textile reinforcements. In view of that, it can be concluded that the 1D model is a valid tool to describe pull-out failure of textile reinforcement concrete specimens. In contrast to the 1D model, 3D models are especially useful to model the presented complex pull-out test with non-symmetrical embedment lengths such that full interaction (i.e. total slip and unloading) between both Short and Long parts of the pull-out specimen can be included simultaneously.

\section{Acknowledgments}

The presented research was funded by the European Community's Seventh Framework Programme under grant agreement NMP2-LA-2009-228663 (TailorCrete) and FORMAS (Homes for Tomorrow). More information about the research projects, TailorCrete and Homes for Tomorrow, can be found at www.tailorcrete.com and www.homesfortomorrow.se, respectively. Special thanks are also extended to the Ministry of Science and Innovation of the Government of Spain (MINECO) for funding Ignasi Fernandez Perez to carry out a research and knowledge exchange at Chalmers University of Technology. 


\section{References}

[1] Orlowsky J, Raupach M. Textile reinforced concrete-from research to application. Cement Wapno Beton. 2011;16(6):323-31.

[2] Mobasher B. Mechanics of fiber and textile reinforced cement composites: CRC Press; 2012.

[3] Hegger J, Goralski C, Kulas C. A pedestrian bridge made of textile reinforced concrete. Schlanke Fußgängerbrücke aus Textilbeton. 2011;106(2):64-71.

[4] Insu-Shell-Projekt LIFE. Life insu-Shell-Projekt • Institut Für Textiltechnik Der Rwth Aachen. 2009.

[5] Ortlepp R, Curbach M. Strengthening of Columns using Textile Reinforced Concrete (TRC). Beton- Und Stahlbetonbau. 2009;104(10):681-9.

[6] Ortlepp R, Schladitz F, Curbach M. Textilbetonverstärkte Stahlbetonstützen. Beton- Und Stahlbetonbau. 2011;106(9):640-8.

[7] Brameshuber W. Test methods and design of Textile Reinforced Concrete Report of RILEM TC 232-TDT: RILEM publications; Not yet available.

[8] Brameshuber W. Report 36: Textile Reinforced Concrete-State-of-the-Art Report of RILEM TC 201-TRC: RILEM publications; 2006.

[9] Zastrau B, Richter M, Lepenies I. On the Analytical Solution of Pullout Phenomena in Textile Reinforced Concrete. Journal of Engineering Materials and Technology. 2003;125(1):38-43.

[10] Krüger M. Vorgespannter textilbewehrter Beton: University of Stuttgart; 2004.

[11] Xu S, Li H. Bond properties and experimental methods of textile reinforced concrete. Journal of Wuhan University of Technology--Materials Science Edition. 2007;22(3):529-32.

[12] Sueki S, Soranakom C, Mobasher B, Peled A. Pullout-slip response of fabrics embedded in a cement paste matrix. Journal of Materials in Civil Engineering. 2007;19(9):718-27.

[13] Ortlepp R, Curbach M, Weiland S. Rehabilitation and strengthening of a hypar concrete shell by textile reinforced concrete. Excellence in Concrete Construction through Innovation: Taylor \& Francis; 2009.

[14] Lorenz E, Ortlepp R. Bond Behavior of Textile Reinforcements-Development of a Pull-Out Test and Modeling of the Respective Bond versus Slip Relation. High Performance Fiber Reinforced Cement Composites 6: Springer; 2012. p. 479-86.

[15] Zhang XB, Aljewifi H, Li J. Failure behaviour investigation of continuous yarn reinforced cementitious composites. Construction and Building Materials. 2013;47(0):456-64.

[16] Richter M, Lepenies I, Zastrau BW. On the influence of the bond behaviour between fiber and matrix on the material properties of textile reinforced concrete. International Symposium of Anisotropic Behaviour of Damaged Materials2002. p. 1-24.

[17] Lundgren K, Kettil P, Hanjari KZ, Schlune H, Roman ASS. Analytical model for the bondslip behaviour of corroded ribbed reinforcement. Structure and Infrastructure Engineering. 2012;8(2):157-69. 
[18] Banholzer B. Bond of a strand in a cementitious matrix. Materials and structures. 2006;39(10):1015-28.

[19] Butler M, Mechtcherine V, Hempel S. Experimental investigations on the durability of fibrematrix interfaces in textile-reinforced concrete. Cement and Concrete Composites. 2009;31(4):221-31.

[20] CEN. Eurocode 2: Design of Concrete Structures: Part 1-1: General Rules and Rules for Buildings: British Standards Institution; 2004.

[21] Lorenz E, Ortlepp R. Basic research on the anchorage of textile reinforcement in cementitious matrix. 9th international symposium on fiber-reinforced polymer reinforcement for concrete structures (FRPRCS-9), book of abstract2009. p. 136.

[22] International Federation for Structural Concrete (fib). fib Bulletin 55: Model Code 2010, First complete draft2010.

[23] Ruiz MF, Muttoni A, Gambarova P. Analytical modeling of the pre-and postyield behavior of bond in reinforced concrete. Journal of Structural Engineering. 2007;133(10):1364-72.

[24] Losberg A. Sprickbildning i kontinuerliga betongbeläggningar och andra betongkonstruktioner, låsta mot rörelser av temperatur och krympning (Cracks in continuous concrete road slabs and other concrete structures locked against movements from temperature and shrinkage). Chalmers University of Technology, Institution of Building Technology 1962;No 607:45.

[25] MATLAB. The MathWorks, Inc; 1994-2013.

[26] TNO Diana. Finite Element Analysis User's Manual - release 9.4.4: TNO; 2011. 